\title{
Combining Structural and Enumerative Techniques for the Validation of Bounded Petri Nets
}

\author{
Rubén Carvajal-Schiaffino, Giorgio Delzanno, and Giovanni Chiola \\ Dipartimento di Informatica e Scienze dell'Informazione \\ Università di Genova \\ via Dodecaneso 35, 16146 Genova \\ \{ruben,giorgio,chiola\}@disi.unige.it
}

\begin{abstract}
We propose a new validation algorithm for bounded Petri Nets. Our method combines state enumeration and structural techniques in order to compute under-approximations of the reachability set and graph of a net. The method is based on two heuristics that exploit properties of T-semiflows to detect acyclic behaviors. T-semiflows also give us an heuristic estimation of the number of levels of the reachability graph we have to keep in memory during forward exploration. This property allows us to organize the space used to store the reachable markings as a circular array, reusing all markings outside a sliding window containing a fixed number of the last levels of the graph. We apply the method to examples taken from the literature $\mathrm{ABC}^{+95} \mathrm{CNI97} \mathrm{NICC} 7$. Our algorithm returns exact results in all the experiments. In some examples, the circular memory allow us to save up to $98 \%$ of memory space, and to scale up to 255 the number of tokens in the specification of the initial marking.
\end{abstract}

\section{Introduction}

Bounded Petri Nets (PNs) are finite-state concurrent systems in which the maximal number of processes (tokens) in any possible state (place) is bounded by a constant. Though decidable, the verification of safety and liveness properties of bounded PNs is a very hard problem in practice. Following the literature in the field STC98 Val98, the techniques used to attack this problem can be distinguished into the following classes.

State Enumeration Techniques. The reachability graph of a finite-state system built using an exhaustive search algorithm $\mathrm{Hol} 88$ is a complete tool for the verification of safety and liveness properties. This technique suffers from the state explosion problem, i.e., the explosion of the size of the reachability graph compared to the size of the specification $\mathrm{BCB}^{+} 90$ Val98. Partial search Hol88 can be used as heuristics to validate large finite-state systems. In general, partial search returns under-approximations of the reachability graph. Therefore,

T. Margaria and W. Yi (Eds.): TACAS 2001, LNCS 2031, pp. 435- 4442001

(C) Springer-Verlag Berlin Heidelberg 2001 
it cannot be used for verification purposes, but only for simulation and testing. When incorporated in search algorithms, efficient data structures like hash tables Hol88, BDDs $\mathrm{BCB}^{+} 90$, and Sharing Trees GG795 represent other important heuristics to alleviate state explosion.

Structural Techniques. While state enumeration is a general-purpose technique for validation of finite-state systems, verification techniques based on structural properties are a distinguishing feature of PNs STC98. These techniques work without explicitly computing the reachability graph. They rely on linear programming (synonymous of efficiency) usually returning approximated answers. For instance, the state equation Rei86 can be used to over-approximate the reachability set of a PN, and thus to verify safety properties STC98. Other techniques like traps can be used to improve the precision of the state equation FMOO .

Our Contribution. In contrast with traditional uses of structural theory, in this paper we investigate the combination of enumerative and structural techniques for validating and debugging systems modeled as bounded PNs. Specifically, we use structural properties as heuristics to guide the search during state exploration. In order to attack state explosion we incorporate our heuristics within a partial search algorithm, and we leave open the possibility of using efficient data structures for storing intermediate results.

More precisely, the algorithm we propose explores part of the state-space of a PN using properties of minimal T-semiflows in order to detect acyclic occurrence sequences without having to search for visited markings. Minimal T-semiflows form a system of generators (the fundamental set) for all the positive integer solutions of the system of equalities

$$
\mathbf{C} \cdot \boldsymbol{x}=0, \mathrm{C} \text { being the token flow matrix. }
$$

To apply our heuristics, we require the fundamental set to be integral, i.e., Tsemiflows must be non-negative integer combinations of minimal T-semiflows. This conditions is satisfied by several case-studies we have found in the literature (see Section 5. Integrality is a new property we introduce on the basis of classical notions of linear programming Sch94. Our algorithm returns an under-approximation of the reachability graph, while automatically measuring the quality of the approximation. Specifically, a flag is raised whenever the returned graph is an equivalent representation of the reachability graph. Thus, in an ideal situation our validation method can also be used as a complete tool for verification. At any moment during the execution, the algorithm works on a sliding window that covers the last levels of the partially constructed graph. The number of levels included in the sliding window is computed statically, using again minimal T-semiflows. This property gives us an estimation of the number of levels of the reachability graph we need to keep in memory during forward exploration. We exploit these information to build the following garbage collection procedure: we organize the main memory as a circular array, and we re-use the memory allocated to all markings outside the window. 
In order to test the applicability of our assumptions and the quality of our heuristics, we run a prototype implementation of the algorithm (without use of dedicate data structures to store the markings) on several examples taken from ABC+95 CM97 MCC.97. Our aim was to check safety properties and compute the reachability set. The preliminary results seem very promising. In some of the examples, we were able to scale up the number of tokens in the initial marking to 255 , and to handle the resulting PN using only 25Mbytes of main memory (within the range of the RAM memory of a personal computer, see Section 5 . Without sliding window the same examples would have required approximatively 1,300 Mbytes of memory, i.e., our heuristics can save up to $98 \%$ of memory space. Finally, we obtained an exact representation of the reachability set in all our experiments, i.e., with our method we were able to verify all safety properties taken into consideration.

Plan of the paper. In Section 2 we recall the main properties of the Structural Theory of Petri Nets. In Section 3 we introduce the notions necessary to our algorithm. In Section 4 we present the heuristics and the validation algorithm. In Section $\square$ we discuss our experimental results. Finally, in Section $\boldsymbol{b}$ and $\boldsymbol{\square}$ we discussed related works and future directions of research, respectively. The extended version of this paper (containing the proofs of all results) is available as technical report $\mathrm{CD}(\mathrm{C} 00$.

\section{Structural Theory for Petri Nets}

Following STC98, a PN $N$ is a tuple $\left\langle P, T\right.$, Pre, Post, $\left.\boldsymbol{m}_{\mathbf{0}}\right\rangle$, where $P$ is the finite set of places, $T$ is the finite set of transitions, Pre and Post are the $|P| \times|T|$ sized, incidence matrices, and $\boldsymbol{m}_{\mathbf{0}}$ is the initial marking. The matrix $\mathbf{C}=$ Post - Pre is called token flow matrix. A marking $\boldsymbol{m}=\left\langle m_{1}, \ldots, m_{n}\right\rangle$ is a vector of natural numbers of dimension $n=|P|$. We will use $\mathbf{0}$ to denote the null vector $\langle 0, \ldots, 0\rangle$. Given two vectors $\boldsymbol{m}=\left\langle m_{1}, \ldots, m_{n}\right\rangle$ and $\boldsymbol{m}^{\prime}=\left\langle m_{1}^{\prime}, \ldots, m_{n}^{\prime}\right\rangle$, we define $\boldsymbol{m} \geq \boldsymbol{m}^{\prime}$ if and only if $m_{i} \geq m_{i}^{\prime}$ for $i: 1, \ldots, n$. Similarly, we can define $\boldsymbol{m}=\boldsymbol{m}^{\prime}$, whereas $\boldsymbol{m}>\boldsymbol{m}^{\prime}$ holds if and only if $\boldsymbol{m} \geq \boldsymbol{m}^{\prime}$ and $\boldsymbol{m} \neq \boldsymbol{m}^{\prime}$.

Occurrence sequences, and Parikh vectors. Let $N$ be a PN with token flow matrix $\mathbf{C}, n$ places, and $m$ transitions $t_{1}, \ldots, t_{m}$. A transition $t \in T$ is enabled at marking $\boldsymbol{m}$ if $\boldsymbol{m} \geq$ Pre[ $[P, t]$, i.e., there are enough tokens to fire $t$. The firing of the transition $t$, namely $\boldsymbol{m} \stackrel{t}{\rightarrow} \boldsymbol{m}^{\prime}$, yields a new marking $\boldsymbol{m}^{\prime}=m+\mathbf{C}[P, t]$. An occurrence sequence from $\boldsymbol{m}$ is a sequence of transitions $\sigma=s_{1} \ldots s_{k}$ such that $\boldsymbol{m} \stackrel{s_{1}}{\rightarrow} \ldots \stackrel{s_{k}}{\rightarrow} \boldsymbol{m}_{\boldsymbol{k}}$. The reachability set is denoted by $\mathcal{R}\left(N, \boldsymbol{m}_{\mathbf{0}}\right)$. The reachability graph is denoted by $\mathcal{G}\left(N, \boldsymbol{m}_{\mathbf{0}}\right)$. The state equation is defined as the system of equalities

$$
\boldsymbol{m}^{\prime}=\boldsymbol{m}_{\mathbf{0}}+\mathrm{C} \cdot \boldsymbol{x}
$$

where $\boldsymbol{m}^{\prime}$ and $\boldsymbol{x}$ are vectors of variables that range over positive integers. The Parikh vector $p_{\sigma}$ associated to a finite occurrence sequence $\sigma$ is defined as follows:

$$
p_{\sigma}=\left\langle O c c_{t_{1}}(\sigma), \ldots, O c c_{t_{m}}(\sigma)\right\rangle,
$$


where $O c c_{t_{i}}(\sigma)=$ number of occurrences of $t_{i}$ in $\sigma$. In the following we will use $\boldsymbol{x}, \boldsymbol{y}, \ldots$ to denote vectors of natural numbers with dimension $=|T|$ (for clarity, always referred to as Parikh vectors).

T-semiflows, and Fundamental Set. An integer vector $\boldsymbol{x}$ of dimension $m$ is called a $T$-flow if and only if

$$
\mathbf{C} \cdot \boldsymbol{x}=0 \text {, where } \mathbf{C} \text { is the token flow matrix. }
$$

The following proposition relates T-flows and cyclic sequences.

Proposition 1 (From STC98). Let $N$ be a $P N$, and let $\boldsymbol{m} \stackrel{\sigma}{\rightarrow} \boldsymbol{m}^{\prime}$. Then, the Parikh vector $p_{\sigma}$ associated to $\sigma$ is a T-flow if and only if $\boldsymbol{m}=\boldsymbol{m}^{\prime}$.

A $T$-semiflow is a T-flow $\boldsymbol{x}$ such that $\boldsymbol{x} \geq \mathbf{0}$. A minimal T-semiflow is a Tsemiflow $\boldsymbol{x}$ such that: the greatest common divider of all its positive components is equal to 1, and there are no T-semiflow $\boldsymbol{y}$ such that the set of non-zero components of $\boldsymbol{y}$ are contained in that of $\boldsymbol{x}$. The fundamental set of T-semiflows, say $\mathcal{F}$, of $N$ is the set of minimal T-semiflows of $N$. The fundamental set can be computed using a variation of the Gaussian elimination method. The number of minimal T-semiflows of a PN $N$ could be exponential in the size of $N$ STC98. T-semiflows enjoy the following properties.

Theorem 1 (From STC98). Let $N$ be a PN with fundamental set $\mathcal{F}=$ $\left\{\boldsymbol{x}_{\mathbf{1}}, \ldots, \boldsymbol{x}_{\boldsymbol{k}}\right\}$. Every $T$-semiflow $\boldsymbol{y}$ can be obtained as a non-negative linear combination with rational coefficients of the minimal T-semiflows, i.e., $\boldsymbol{y}=c_{1} \boldsymbol{x}_{\mathbf{1}}+$ $\ldots+c_{k} \boldsymbol{x}_{\boldsymbol{k}}$, where $\boldsymbol{x}_{\boldsymbol{i}} \in \mathcal{F}, c_{i} \in \mathbf{Q}$, and $c_{i} \geq 0$ for $i: 1, \ldots, k$.

In the following we will call $\operatorname{Lin}_{\mathbf{Q}^{+}}(\mathcal{F})\left(\operatorname{Lin}_{\mathrm{Z}^{+}}(\mathcal{F})\right)$ the set of vectors obtained as non-negative linear combinations with rational (integer) coefficients of vectors in $\mathcal{F}$. From Theorem II and Prop. II we obtain the following corollary.

Corollary 1 (Cycle $\Rightarrow$ T-semiflow STC98). Let $N$ be a PN, and let $\boldsymbol{m} \stackrel{\sigma}{\rightarrow}$ $\boldsymbol{m}^{\prime}$. If $\boldsymbol{m}=\boldsymbol{m}^{\prime}$, i.e., $\sigma$ is a cycle in $\mathcal{G}(N, \boldsymbol{m})$, then $p_{\sigma} \in \operatorname{Lin}_{\mathbf{Q}^{+}}(\mathcal{F})$.

The reverse implication might not hold. A counterexample of a PN in which a T-semiflow is not realizable (all paths denoted by the T-semiflow are not valid occurrence sequences) is given by Reisig in Rei86. Note that for Free-choice PNs DE95 minimal T-semiflows are always realizable. Unfortunately, this class does not permit to model interesting examples of mutual-exclusion algorithms.

\section{Towards the Combination with State Enumeration}

Our starting point consists in a reformulation of the standard exhaustive search algorithm using Parikh vectors. The unique goal of this preliminary step is to simplify the integration of our structural heuristics in the enumerative approach. 
Algorithm $\operatorname{ES}(N, \mathcal{P})$ : Boolean.

$N=\left\langle P, T\right.$, Pre, Post, $\left.\boldsymbol{m}_{\mathrm{O}}\right\rangle: \mathrm{PN}$;

$\mathcal{P}$ : the safety property;

Old: $=\emptyset$;

New: $=\left\{m_{0}\right\}$;

while (New nonempty) do $m=$ element from New;

if $\operatorname{not}(\mathcal{P}(m))$ then return(false);

for every $\mathrm{t}_{i} \in T$ enabled at $\boldsymbol{m}$ do

$m^{\prime}=m+\mathrm{C}\left[P, \mathbf{t}_{i}\right]$

if $\left(m^{\prime} \notin\right.$ Old $\cup$ New $)$ then add $m^{\prime}$ to New:

endf;

add $m$ to Old;

delete $m$ from New:

endw;

return(true).
Algorithm DES $(N, \mathcal{P})$ : Boolean.

$N=\left\langle P, T\right.$, Pre, Post, $\left.\boldsymbol{m}_{\mathbf{0}}\right\rangle:$ PN;

$\mathcal{P}$ : the safety property;

$y_{0}:=\mathbf{0}$

OLD $:=\emptyset$;

New $:=\left\{\boldsymbol{y}_{0}\right\}$

while (NEw nonempty) do

$y=$ element from NEW;

if $\operatorname{not}(\mathcal{P}(M(y)))$ then return(false);

for every $\mathbf{t}_{\mathbf{i}} \in T$ enabled at $M(\boldsymbol{y})$ do $\boldsymbol{y}^{\prime}=\boldsymbol{y}\left[y_{i}:=y_{i}+1\right]$;

if $\left(M\left(y^{\prime}\right) \notin M(\right.$ OLD $\cup$ NEW $\left.)\right)$ then add $\boldsymbol{y}^{\prime}$ to NEw;

endf;

add $y$ to OLD;

delete $\boldsymbol{y}$ from NEW;

endw;

return(true).

Fig. 1. Two formulations of the Type 1 Reachability Algorithm for PNs.

\subsection{An Encoding Based on Parikh Vectors}

Let $N$ be a PN with $n$ places, $m$ transitions, token flow matrix $\mathbf{C}$, and initial marking $\boldsymbol{m}_{\mathbf{0}}$. Following Hol88, the exhaustive search procedure ES (exhaustive search) of Fig. II builds the complete reachability set (graph) storing the set of visited markings in the variable Old. The procedure ES can be reformulated using a representation of a reachable marking $\boldsymbol{m}$ via the Parikh vector $p_{\sigma}$ associated to the path $\sigma$ such that $\boldsymbol{m}_{\mathbf{0}} \stackrel{\sigma}{\rightarrow} \boldsymbol{m}$. In fact, from the state equation we know that

$$
\boldsymbol{m}=\boldsymbol{m}_{\mathbf{0}}+\mathbf{C} \cdot p_{\sigma} .
$$

A Parikh vector $\boldsymbol{x}$ can be used as a concise representation for all realizable paths $\sigma$ starting from $\boldsymbol{m}_{\mathbf{0}}$ such that $p_{\sigma}=\boldsymbol{x}$. Given a Parikh vector $\boldsymbol{y}$ we define the marking $M(\boldsymbol{y})$ associated to $\boldsymbol{y}$ as

$$
M(\boldsymbol{y})=\boldsymbol{m}_{\mathbf{0}}+\mathrm{C} \cdot \boldsymbol{y} .
$$

Note that $M\left(\boldsymbol{y}_{\mathbf{0}}\right)=\boldsymbol{m}_{\mathbf{0}}$ whenever $\boldsymbol{y}_{\mathbf{0}}=\mathbf{0}$. Furthermore, given a set of Parikh vectors $\mathrm{S}$ we define

$$
M(\mathrm{~S})=\{\boldsymbol{m} \mid \boldsymbol{m}=M(\boldsymbol{y}), \boldsymbol{y} \in \mathrm{S}\} .
$$

Using the mapping $M(\cdot)$, we can reformulate the forward reachability algorithm representing explicitly the Parikh vectors underlying every marking, as shown in the dual exhaustive search procedure DES (dual exhaustive search) of Fig. II In the algorithm DES (the skeleton of ES), firing a transition $t_{i}$ enabled at $M(\boldsymbol{y})$ modifies a vector $\boldsymbol{y}=\left\langle y_{1}, \ldots, y_{n}\right\rangle$ as follows

$$
\boldsymbol{y}\left[y_{i}:=y_{i}+1\right]=\left\langle y_{1}, \ldots, y_{i-1}, y_{i}+1, y_{i+1}, \ldots, y_{n}\right\rangle \text {. }
$$


Suppose we run the two algorithms of Fig. II in parallel, then the following properties hold at any step: $\boldsymbol{m}^{\prime}=M\left(\boldsymbol{y}^{\prime}\right)$, Old $=M($ Old $)$, and New $=M(\mathrm{New})$. From now on, we will use the algorithm DES as a platform to include the set of heuristics based on properties of T-semiflows described in the following section.

\subsection{Sufficient Conditions for Detecting Acyclic Behaviors}

As shown next, the contraposed form of Cor. 11 of Section 2 can be used to devise sufficient conditions for detecting acyclic occurrence sequences without having to search for visited markings.

Corollary 2 (Not-T-semiflow $\Rightarrow$ Not-Cycle). Let $N$ be a PN, and let $\boldsymbol{m} \stackrel{\sigma}{\rightarrow}$ $\boldsymbol{m}^{\prime}$. If $p_{\sigma} \notin \operatorname{Lin}_{\mathbf{Q}^{+}}(\mathcal{F})$ then $\sigma$ is a not a cycle in $\mathcal{G}(N, \boldsymbol{m})$.

This property goes well together with our formulation of the forward reachability algorithm using Parikh vectors. Before entering in more details, let us first analyze the cost needed to check the condition $p_{\sigma} \notin \operatorname{Lin}_{\mathbf{Q}^{+}}(\mathcal{F})$ of Cor. $\mathbf{Z}$ To test this condition, we must solve a linear problem with rational solutions (polynomial in the size of $\mathcal{F}$ ). Are there more efficient sufficient conditions (e.g. linear in $\mathcal{F}$ ) we can use? To answer this question, let us introduce the following new notion.

Definition 1 (Integral Fundamental Set). We say that the fundamental set $\mathcal{F}$ is integral whenever $\boldsymbol{x} \in \operatorname{Lin}_{\mathbf{Q}^{+}}(\mathcal{F})$ implies that $\boldsymbol{x} \in \operatorname{Lin}_{\mathbf{Z}^{+}}(\mathcal{F})$, i.e. all T-semiflows can be computed using non-negative combinations with integer coefficients.

Under the assumption that $\mathcal{F}$ is integral, the following theorem can be used as a sufficient condition for detecting acyclic behaviors.

Theorem 2 (Sufficient Condition for Not-T-semiflow). Let $N$ be a $P N$ with $m$ transitions, and integral fundamental set $\mathcal{F}=\left\{\boldsymbol{x}_{1}, \ldots, \boldsymbol{x}_{\boldsymbol{k}}\right\}$, where $\boldsymbol{x}_{\boldsymbol{i}}=$ $\left\langle x_{i, 1}, \ldots, x_{i, m}\right\rangle$. Furthermore, let $\boldsymbol{m} \stackrel{\sigma}{\rightarrow} \boldsymbol{m}^{\prime}$, and $p_{\sigma}=\left\langle y_{1}, \ldots, y_{m}\right\rangle$ be the Parikh vector associated to $\sigma$. If for all $i: 1, \ldots, k$ there exists $j \in\{i, \ldots, m\}$ such that $x_{i, j}>y_{j}$, then for all non-empty subpath $\sigma^{\prime}$ of $\sigma, p_{\sigma}^{\prime} \notin \operatorname{Lin} \mathbf{Q}^{+}(\mathcal{F})$.

The cost of checking the condition of Theorem $D$ is linear in the cardinality of $\mathcal{F}$. The cardinality of $\mathcal{F}$ is potentially exponential in the size of $N$, but it is often linear in practice (see Section $\mathbf{n}$. As a remark, note the difference between the hypotheses of Theorem $\boldsymbol{\nabla}$ and those of Prop I namely $\mathbf{C} \cdot p_{\sigma} \neq \mathbf{0}$. If Theorem $\boldsymbol{2}$ holds, then all subpaths contained in the path $\sigma$ from $\boldsymbol{m}$ to $\boldsymbol{m}^{\prime}$ are acyclic. Contrary, if $\mathbf{C} \cdot p_{\sigma} \neq 0$, then we deduce that only the paths from $\boldsymbol{m}$ to $\boldsymbol{m}^{\prime}$ are acyclic. However, it is easy to build a Petri Net for which there exist three markings $\boldsymbol{m}, \boldsymbol{m}^{\prime}$ and $\boldsymbol{m}^{\prime \prime}$ such that $\boldsymbol{m} \stackrel{\sigma_{2}}{\rightarrow} \boldsymbol{m}^{\prime \prime} \stackrel{\sigma_{2}}{\rightarrow} \boldsymbol{m}^{\prime}, \mathbf{C} \cdot p_{\sigma_{1}+\sigma_{2}} \neq \mathbf{0}$, and $\mathbf{C} \cdot p_{\sigma_{2}}=\mathbf{0}$. 


\subsection{Checking the Integrality of $\mathcal{F}$}

To check the integrality of the fundamental set, we can use the notion of total unimodularity Sch94. A matrix A with integer coefficients is totally unimodular if every subdeterminant of $\mathbf{A}$ is 0,1 or -1 . From Sch94, we know that if $\mathbf{A}$ is totally unimodular, then the extreme points of the set of solutions of $\mathbf{A} \cdot \boldsymbol{x}=\boldsymbol{b}$ are integer numbers for any vector $\boldsymbol{b}$. Furthermore, to check the total unimodularity of $\mathcal{F}$, we can use the following (polynomial-time) criterion on the matrix with minimal T-semiflows as rows.

Theorem 3 (From Sch94). Let A be matrix with two non-zero coefficient in each column. A is totally unimodular iff its rows can be split into two classes such that for each column: if the nonzero in the column have the same sign then they are in different classes, and if they have opposite signs they are both in the same class.

Thus, if $\mathcal{F}$ forms a totally unimodular matrix, then $\boldsymbol{x} \in \operatorname{Lin}_{\mathbf{Q}^{+}}(\mathcal{F})$ if and only if $\boldsymbol{x} \in \operatorname{Lin}_{\mathbf{Z}^{+}}(\mathcal{F})$. Perhaps surprisingly, several examples taken from the literature satisfy the integrality requirement on $\mathcal{F}$. We will turn back to this point in Section 5

\section{Partial Search with Structural Heuristics}

We come now to the definition of our partial search algorithm. Basically, the idea is to replace the core of the reachability algorithm DES of Fig. II with two heuristics selected on the basis of a preliminary comparison of Parikh vectors with minimal T-semiflows. The first heuristics exploits Theorem 2 to add markings to the set of visited states. The second heuristics applies sufficient conditions to localize the search for back-edges in the reachability graph. A Boolean flag (we will call complete) is used to estimate the quality of the approximation computed by the heuristics. The resulting partial search PS algorithm is shown in Fig. 2 To explain it in detail, in the rest of the section we will use the predicate SFC defined as

$$
\mathrm{SFC}(\boldsymbol{y}) \doteq \text { for all } \boldsymbol{x}=\left\langle x_{1}, \ldots, x_{m}\right\rangle \in \mathcal{F} \text { exists } i \in\{i, \ldots, m\} \text { s.t. } y_{i}<x_{i} .
$$

to denote the comparison between a Parikh vector $\boldsymbol{y}=\left\langle y_{1}, \ldots, y_{m}\right\rangle$ and the minimal T-semiflows of $\mathcal{F}$. Now, let $\boldsymbol{y}^{\prime}$ be the new Parikh vector generated during the execution of forward reachability, and let OLD and NEw denote the set of visited markings.

The First Structural Heuristics. Suppose SFC $\left(\boldsymbol{y}^{\prime}\right)$ holds. From Theorem $\boldsymbol{\nabla}$ and Cor. $\boldsymbol{2}$ we can deduce that the marking $M\left(\boldsymbol{y}^{\prime}\right)$ is not present in all paths $\sigma$, $p_{\sigma}=\boldsymbol{y}^{\prime}$, going from $\boldsymbol{m}_{\mathbf{0}}$ to $M\left(\boldsymbol{y}^{\prime}\right)$. Under this hypothesis, our heuristics is defined as follows: without further checks on OLD we instruct the algorithm to immediately add $\boldsymbol{y}^{\prime}$ to NEw. The advantage of the heuristics is that we avoid the cost of searching for (a possible occurrence of) $M\left(\boldsymbol{y}^{\prime}\right)$ in the whole 
graph. The drawback is that it could introduce redundant markings. In fact, the marking $M\left(\boldsymbol{y}^{\prime}\right)$ may occur in paths unrelated to $\boldsymbol{y}^{\prime}$ (not captured by Cor. 2. This fact does not influence the termination of the resulting algorithm, as stated in Theorem 4 We postpone the practical evaluation of the first heuristics to Section 5

The Second Structural Heuristics. Suppose that SFC $\left(\boldsymbol{y}^{\prime}\right)$ does not hold. Then, there exists some $\boldsymbol{x} \in \mathcal{F}$ such that $\boldsymbol{y}^{\prime} \geq \boldsymbol{x}$. In other words, all paths $\sigma$ such that $p_{\sigma}=\boldsymbol{y}^{\prime}$ contain a subpath that is a minimal T-semiflow. Furthermore, since by definition $\mathbf{C} \cdot \boldsymbol{x}=0$, if we apply the state equation we obtain that

$$
M\left(\boldsymbol{y}^{\prime}\right)=\boldsymbol{m}_{\mathbf{0}}+\mathrm{C} \cdot\left(\boldsymbol{y}^{\prime}-\boldsymbol{x}\right) .
$$

Our idea is to use the normalized Parikh vector $\boldsymbol{y}^{\prime}-\boldsymbol{x}$ to guide the search for a marking $\boldsymbol{m} \in$ OLD such that $\boldsymbol{m}=M\left(\boldsymbol{y}^{\prime}\right)$. Formally, let the rank of a Parikh vector $\boldsymbol{y}$ be defined as

$$
\operatorname{rank}\left(\left\langle y_{1}, \ldots, y_{n}\right\rangle\right)=y_{1}+\ldots+y_{n} .
$$

Furthermore, given a set of Parikh vectors $\mathrm{S}$, let the $k$-th level of $\mathrm{S}$ be defined as

$$
\mathrm{S}[k]=\{\boldsymbol{y} \mid \boldsymbol{y} \in \mathrm{S}, \operatorname{rank}(\boldsymbol{y})=k\} .
$$

Then, if SFC $\left(\boldsymbol{y}^{\prime}\right)=$ false, we first search for a marking $\boldsymbol{m}$ such that $\boldsymbol{m}=M\left(\boldsymbol{y}^{\prime}\right)$ in all levels OLD $\left[\operatorname{rank}\left(\boldsymbol{y}^{\prime}-\boldsymbol{x}\right)\right]$ with $\boldsymbol{x} \in \mathcal{F}$ and $\boldsymbol{y}^{\prime} \geq \boldsymbol{x}$. If we find the node we draw a back-edge. The edge will be part of a cycle. If the previous local search fails, we discharge the vector $\boldsymbol{y}^{\prime}$, while setting the Boolean flag complete to false. This way, we inform the user that the algorithm is computing an underapproximation of the reachability graph. Basically, we substitute the full termination test $\boldsymbol{y} \in$ OLD of the algorithm DES of Fig. I with a sufficient condition. If the flag complete is true when the algorithm terminates the exploration of the state space, then the resulting reachability graph is exact. The following theorem formalizes these properties.

Theorem 4. Let $N$ be a bounded $P N$ with integral fundamental set $\mathcal{F}, \mathcal{P}$ a safety property and let $C$ be the value of the flag complete when the algorithm PS of Fig. 0 returns. Then, (1) the computation of $\operatorname{PS}(N, \mathcal{P})$ always terminates (returning true or false); (2) if $\operatorname{PS}(N, \mathcal{P})=$ true and $C=$ true, then $\mathcal{P}$ holds for $N$; (3) if $\operatorname{PS}(N, \mathcal{P})=$ false, then $\mathcal{P}$ does not hold for $N$.

The second heuristics gives us a bound on the number of levels we have to keep in memory during the exploration of the reachability graph. The bound WS (window size) is the maximum between the ranks of the minimal T-semiflows in $\mathcal{F}$, namely

$$
W S=\max \{\operatorname{rank}(\boldsymbol{x}) \mid \boldsymbol{x} \in \mathcal{F}\} .
$$

Thus, our algorithm works only on a window of dimension WS that covers the last levels of the current reachability set. We will present a memory management based on this property in the next section. 


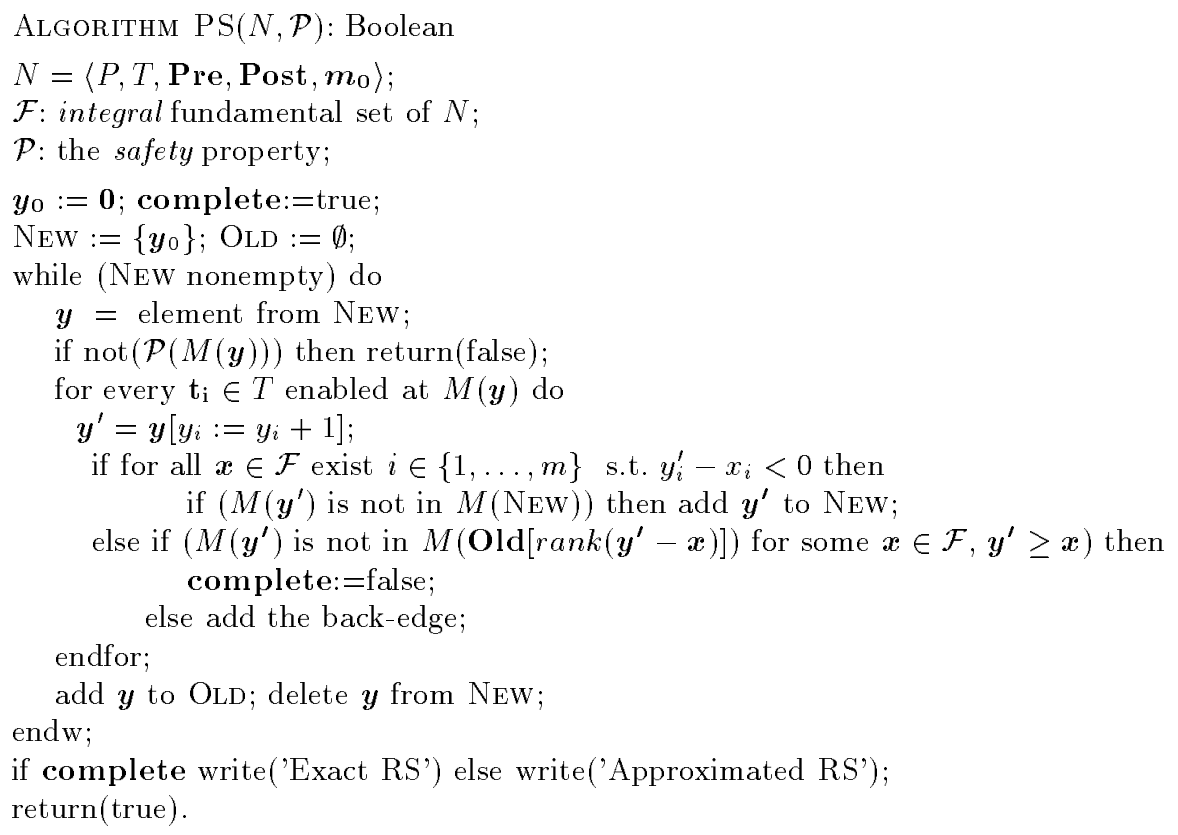

Fig. 2. A Type 2 Reachability Algorithm.

\section{$5 \quad$ Experimental Results}

We have implemented a prototype version of the algorithm DFR of Fig. $\mathbf{2}$ borrowing the graphical interface and the library for computing structural properties from GreatSPN CFGR95, and using the following specialized memory management.

\subsection{Organizing the Memory as a Circular Array}

We consider PNs where transitions can be fired at most 255 times. We organize the available memory (RAM + swap area) as a circular array, where each slot in the array contains $m$ bytes and stores a Parikh vector $(m=$ number of transitions). Our representation does not depend on the bound on the number of tokens in the places. If $T M$ is the size of allocated memory in bytes, the number of available slots $A S$ is then $A S=T M / m$. Furthermore, if $N S$ is the number or reachable vectors, then the virtual memory required to store them is $M S=N S * m$ bytes. A table maintains the initial and final address of the set of Parikh vectors of each level. Each level is stored as an ordered list. A sliding window covering the last WS levels of the reachability graph moves around the circular array (we defined $W S$ in the previous section). The global size of the sliding window is the sum of the number of states in each of its levels. By construction of PS, we can always reuse the states outside the window in successive 


\begin{tabular}{|c|c|c|c|}
\hline CASE-STUDY & \begin{tabular}{|l|l|}
$\mathrm{T}$ & $\mathrm{P}$ \\
\end{tabular} & \begin{tabular}{l|l}
$\mathrm{SF}$ & $\mathrm{ET}$ \\
\end{tabular} & 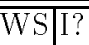 \\
\hline Kanban CM97 & \begin{tabular}{|l|l|l|l}
16 & 16 \\
\end{tabular} & $50.01 \mathrm{~s}$ & $8 \sqrt{ }$ \\
\hline Flexible Manufacturing System (FMS) CII97 & \begin{tabular}{|l|l|}
20 & 22 \\
\end{tabular} & 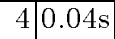 & \begin{tabular}{l|l}
13 & $\sqrt{ }$ \\
\end{tabular} \\
\hline Multipoll MCC97 & \begin{tabular}{|l|l|}
21 & 18 \\
\end{tabular} & $\begin{array}{lll}8 & 0.06 \mathrm{~s} \\
\end{array}$ & $5 \sqrt{ }$ \\
\hline \begin{tabular}{|c|c|c|} 
Central Server Model (CSM) & ABC. 76 pp. 154 \\
\end{tabular} & \begin{tabular}{|l|l|}
13 & 14 \\
\end{tabular} & \begin{tabular}{l|l|l|l|l}
4 & $0.03 \mathrm{~s}$ \\
\end{tabular} & $5 \sqrt{ }$ \\
\hline Readers-Writers $\mathrm{ABC}^{+} 95$ Fig. 11 pp. 17 & \begin{tabular}{|l|l|}
7 & 7 \\
\end{tabular} & \begin{tabular}{l|l|l|}
2 & $0.02 \mathrm{~s}$ \\
\end{tabular} & \begin{tabular}{l|l}
4 & $\sqrt{ }$ \\
\end{tabular} \\
\hline $2 \times 2$ Mesh ABC $^{+} 95$ Fig. 130 pp. 256 & \begin{tabular}{|l|l|}
32 & 32 \\
\end{tabular} & \begin{tabular}{l|l|l|}
8 & $0.07 \mathrm{~s}$ \\
\end{tabular} & \begin{tabular}{l|l}
5 & $\sqrt{ }$ \\
\end{tabular} \\
\hline
\end{tabular}

Fig. 3. Profile of the case-studies: $\mathrm{T}=$ number of transitions; $\mathrm{P}=$ number of places; $\mathrm{SF}($ size of $\mathcal{F})=$ number of minimal T-semiflows; $\mathrm{ET}=\mathrm{CPU}$ execution time to compute $\mathcal{F}$ using GSPN on a Pentium $133 \mathrm{Mhz}$; WS=size of the sliding window; $\mathrm{I} ?=$ is the fundamental set integral?

iterations. An overflow exception $O F$ is raised as soon as the algorithm adds a slot of the last level of the window to its first level (i.e. the window covers all memory). $N R$ will indicates the number of times the last slot of the sliding window goes beyond the rightmost limit of the array $(N R=0$ means $M S \leq T M)$. Finally, the ratio $R$ defined as $1-T M / M S$ give us an estimation of the saving of memory occupancy we obtain with our heuristics.

\subsection{Practical Evaluation}

At this stage of our work, the purposes of the experiments were: (1) testing the applicability of the assumptions under which the algorithm works (the existence of an integral fundamental set); (2) testing the quality and efficiency of our heuristics; (3) testing the scalability of the specialized memory management.

Applicability. To make the tests more interesting, we considered models of concurrent and productions systems taken from ABC+95 C.M97 MCC.97. Furthermore, in order to study the scalability of our approach we restricted ourselves to consider systems with parametric initial markings, where the parameter is the number of initial tokens in some given places of the net. For these examples, we were interested in computing the set of reachable states, so as to prove safety properties like mutual exclusion. As shown in Fig. 3 most of the examples in $A B C+95$ CM97 MCCO7 with the previous characteristics turned out to have integral fundamental set. We computed $\mathcal{F}$ using the structural library of GSPN within negligible execution times (see again Fig. B. We remark that only the Kanban system of CVM7 is a free choice net, all the other examples heavily rely on the use of semaphores.

Quality and Efficiency. In order to test the quality and efficiency of our heuristics, we compared the execution times of our prototype with those of GreatSPN CFGR95, one of the more efficient tools for the generation of the reachability graph of a PN. We performed all experiments on a Pentium with a clock speed 


\begin{tabular}{|c|c|c|c|c|c|c|}
\hline CASE-STUDY & NT & ET-Prot & $\overline{\text { NS-Prot }}$ & $\mathrm{CF}$ & ET-GSPN & NS-GSPN \\
\hline \multirow[t]{4}{*}{ Kanban } & 2 & $1.530 \mathrm{~s}$ & 4,600 & true & $0.860 \mathrm{~s}$ & 4600 \\
\hline & $\overline{4}$ & $229.070 \mathrm{~s}$ & 454,475 & true & $158.700 \mathrm{~s}$ & 454,475 \\
\hline & 5 & $1464.270 \mathrm{~s}$ & $2,546,432$ & true & $\sqrt{ }$ & $\sqrt{ }$ \\
\hline & 6 & $\sqrt{ }$ & $\sqrt{ }$ & - & & \\
\hline \multirow[t]{3}{*}{ FMS } & 2 & $1.270 \mathrm{~s}$ & 3,444 & true & $0.460 \mathrm{~s}$ & $\overline{3,444}$ \\
\hline & $\overline{4}$ & $249.170 \mathrm{~s}$ & 438,600 & true & $117.770 \mathrm{~s}$ & 438,600 \\
\hline & 5 & $\sqrt{ }$ & $\sqrt{ }$ & - & $\sqrt{ }$ & $\sqrt{ }$ \\
\hline \multirow[t]{4}{*}{ Multipoll } & 2 & $5.210 \mathrm{~s}$ & 11,328 & true & $2.190 \mathrm{~s}$ & 11,328 \\
\hline & $\overline{4}$ & $56.280 \mathrm{~s}$ & 106,280 & true & $27.030 \mathrm{~s}$ & 106,280 \\
\hline & 9 & $1164.750 \mathrm{~s}$ & $1,943,160$ & true & $\sqrt{ }$ & $\sqrt{ }$ \\
\hline & 10 & $\sqrt{ }$ & $\sqrt{ }$ & - & & \\
\hline \multirow[t]{2}{*}{ Mesh } & 2 & $178.870 \mathrm{~s}$ & 200,544 & true & $46.150 \mathrm{~s}$ & 200,544 \\
\hline & 3 & $\sqrt{ }$ & $\sqrt{ }$ & - & $\sqrt{ }$ & $\sqrt{ }$ \\
\hline \multirow[t]{5}{*}{ CSM } & $\overline{2}$ & $0.020 \mathrm{~s}$ & 76 & true & $0.010 \mathrm{~s}$ & 76 \\
\hline & 32 & $23.180 \mathrm{~s}$ & 95,876 & true & $27.920 \mathrm{~s}$ & 95,876 \\
\hline & $\overline{75}$ & $311.530 \mathrm{~s}$ & $1,170,704$ & true & $538.450 \mathrm{~s}$ & $1,170,704$ \\
\hline & 115 & $1156.240 \mathrm{~s}$ & $4,162,544$ & true & $\sqrt{ }$ & $\sqrt{ }$ \\
\hline & 116 & $\sqrt{ }$ & $\sqrt{ }$ & - & & \\
\hline \multirow[t]{5}{*}{ Reader-Writers } & 4 & $0.030 \mathrm{~s}$ & 90 & true & $0.010 \mathrm{~s}$ & 90 \\
\hline & 32 & $7.170 \mathrm{~s}$ & 64,889 & true & $10.250 \mathrm{~s}$ & 64,889 \\
\hline & 62 & $94.350 \mathrm{~s}$ & 762,384 & true & $175.300 \mathrm{~s}$ & 762,384 \\
\hline & 114 & $1069.020 \mathrm{~s}$ & $7,927,295$ & true & $\sqrt{ }$ & $\sqrt{ }$ \\
\hline & 115 & $\sqrt{ }$ & $\sqrt{ }$ & - & & \\
\hline
\end{tabular}

NT=Number of Tokens in the initial marking;

ET $=$ CPU Execution Time on a Pentium 133Mhz;

NS=Number of reachable markings;

$\mathrm{CF}=$ value of the Complete Flag when PS returns;

$\sqrt{ }=$ memory overflow;

- Prot=executed on our prototype;

GSPN=executed on GreatSPN CFGR9.5

Fig. 4. First serie of experimental evaluations.

of $133 \mathrm{Mhz}$, RAM memory of 32 Mbytes, and swap area of 34 Mbytes, allocating a priori 55 Mbytes of memory to store the reachability set. The table in Fig. 1 summarizes the results of a first serie of experiments. Surprisingly, the algorithm returned an exact representation (without redundancies) of the reachability set in all the examples (and different values for the parameter=number of tokens in the initial marking). In all the experiments of Fig. 4 we never had to exploit the circularity of our memory organization: 55 Mbytes where enough to store the reachability set. The cost of our heuristics and of the localized search turned out to be comparable to that of the efficient search of GreatSPN (despite the fact that GreatSPN makes also use of simplification rules). However, on examples like Reader-Writers GreatSPN was not able to compute the reachability graph for nets with more than 62 tokens in the initial marking (as indicated by the over- 


\begin{tabular}{|r|r|r|r|r|r|r|r|r|}
\hline \hline \multicolumn{8}{|c|}{ Readers-Writers (No. trans. m=7) executed on our prototype } \\
\hline NT & TM & NS & MS & NR & R & ET & CF & OF \\
\hline 255 & $45 \mathrm{Mb}$ & $185,977,536$ & $1,302 \mathrm{Mb}$ & 28 & $96 \%$ & $27,981 \mathrm{~s}$ & true & \\
\hline 255 & $35 \mathrm{Mb}$ & $185,977,536$ & $1,302 \mathrm{Mb}$ & 37 & $97 \%$ & $27,996 \mathrm{~s}$ & true & \\
\hline 255 & $25 \mathrm{Mb}$ & $185,977,536$ & $1,302 \mathrm{Mb}$ & 52 & $98 \%$ & $27,991 \mathrm{~s}$ & true & \\
\hline 255 & $21 \mathrm{Mb}$ & $66,252,650$ & $463 \mathrm{Mb}$ & 22 & $95 \%$ & $9,719 \mathrm{~s}$ & true & $\sqrt{ }$ \\
\hline 128 & $45 \mathrm{Mb}$ & $12,440,544$ & $87.1 \mathrm{Mb}$ & 1 & $48 \%$ & $1,723 \mathrm{~s}$ & true & \\
\hline 128 & $35 \mathrm{Mb}$ & $12,440,544$ & $87.1 \mathrm{Mb}$ & 2 & $60 \%$ & $1,722 \mathrm{~s}$ & true & \\
\hline 128 & $25 \mathrm{Mb}$ & $12,440,544$ & $87.1 \mathrm{Mb}$ & 3 & $71 \%$ & $1,721 \mathrm{~s}$ & true & \\
\hline 128 & $15 \mathrm{Mb}$ & $12,440,544$ & $87.1 \mathrm{Mb}$ & 5 & $82 \%$ & $1,722 \mathrm{~s}$ & true & \\
\hline 128 & $5 \mathrm{Mb}$ & $12,440,544$ & $87.1 \mathrm{Mb}$ & 17 & $94 \%$ & $1,723 \mathrm{~s}$ & true & \\
\hline 128 & $3 \mathrm{Mb}$ & $5,631,404$ & $40 \mathrm{Mb}$ & 13 & $92 \%$ & $766.6 \mathrm{~s}$ & true & $\sqrt{ }$ \\
\hline 64 & $1 \mathrm{Mb}$ & 860,145 & $6 \mathrm{Mb}$ & 6 & $83 \%$ & $108.3 \mathrm{~s}$ & true & \\
\hline 64 & $500 \mathrm{~Kb}$ & 860,145 & $6 \mathrm{Mb}$ & 12 & $91 \%$ & $108.5 \mathrm{~s}$ & true & \\
\hline 64 & $250 \mathrm{~Kb}$ & 169,728 & $1.2 \mathrm{Mb}$ & 4 & $25 \%$ & $19.6 \mathrm{~s}$ & true & $\sqrt{ }$ \\
\hline 32 & $300 \mathrm{~Kb}$ & 64,889 & $455 \mathrm{~Kb}$ & 1 & $34 \%$ & $7.33 \mathrm{~s}$ & true & \\
\hline 32 & $75 \mathrm{~Kb}$ & 64,889 & $455 \mathrm{~Kb}$ & 6 & $83 \%$ & $7.38 \mathrm{~s}$ & true & \\
\hline 32 & $50 \mathrm{~Kb}$ & 23,099 & $162 \mathrm{~Kb}$ & 3 & $69 \%$ & $2.38 \mathrm{~s}$ & true & $\sqrt{ }$ \\
\hline \hline
\end{tabular}

$\mathrm{NT}=$ number of tokens in the initial marking;

TM=total allocated memory;

$\mathrm{NS}=$ number of reachable states;

$\mathrm{MS}=\mathrm{NS} * \mathrm{~m}$;

$\mathrm{NR}=$ number of rounds in the circular memory;

$\mathrm{R}=1-(\mathrm{TM} / \mathrm{MS}$ ) (saving ratio in pct);

$\mathrm{ET}=\mathrm{CPU}$ execution time on a Pentium, 133Mhz;

$\mathrm{CF}=$ complete flag;

$\mathrm{OF}=$ overflow flag.

Fig. 5. Second serie of experimental evaluations.

flow flag $\checkmark)$. This fact is due to the overhead of a more sophisticated encoding of markings and to the organization of visited markings as a tree structure Chr89 (trade-off between efficient search operations and memory requirements). Both our prototype and GreatSPN store the edges of the reachability graph on disk.

Scalability. In order to test the scalability of our method, we performed a second serie of experiments in which we successively reduced the quantity of memory allocated for storing the reachability set. The aim was to test the efficacy of the circular implementation of the memory. The results were quite surprising. For instance, as shown in Fig. 1 we were able to scale up to 255 the number of tokens in the initial marking of Readers-Writers. In this case the net has approximatively 185 millions of reachable states. It would take approximatively 1300 Mbytes of memory to store the entire reachability set. With our heuristics, we were able to run the example using only 25 Mbytes of memory, hence saving $98 \%$ of memory space. The memory manager returned an overflow exception (indi- 
cated again with $\checkmark$ ) when we tried to use $21 \mathrm{Mb}$ of memory. Furthermore, for an initial marking with 128,64 , and 32 tokens we were able to compute the reachability set saving (approximatively) $94 \%$ (TM=5Mbytes), $92 \%$ (TM=0.5Mbytes), and $84 \%(\mathrm{TM}=75 \mathrm{~Kb})$ of memory space, respectively. We obtained similar results for the CSM example. The results on the other examples were less appealing, though we also managed to scale up FMS to an initial marking with 5 tokens. However, we believe that more results will be obtained by using efficient data structures to store sets of markings.

\section{Related Works}

As mentioned in the introduction, structural techniques are traditionally used to compute over-approximations of the reachability set, see e.g. STCG8. In FVI01, traps are used to improve the quality of the approximation. Place invariants can also be used to over-approximate the reachability set. Place invariants are the dual notion of T-semiflows, i.e., the solution of the system $\boldsymbol{y} \cdot \mathbf{C}=0$. Let $\mathbf{P}$ be the matrix of minimal P-semiflows. As shown in STC98, the solution of the equation $\mathbf{P} \cdot \boldsymbol{x}=\mathbf{P} \cdot \boldsymbol{m}_{\mathbf{0}}$ over-approximates the set of solutions of the state equation $\boldsymbol{m}=\boldsymbol{m}_{\mathbf{0}}+\mathrm{C} \cdot \sigma$, i.e., over-approximates the reachability set. Contrary, in our approach we have used T-semiflows to find under-approximations (useful for debugging) and to derive conditions to establish the quality of the approximation. Furthermore, differently from EM00 STC98, our approach is incorporated in state enumeration. We are not aware of other approaches where T-semiflows are used for under-approximating the reachability set. In MC99, Miner and Ciardo use MDDs (Multi-valued Decision Diagrams) to store the reachability set; whereas, Pastor et al. PCP99 use P-invariants (semiflows) to improve a BDDbased encoding of the reachability set. Other compact data structures (like Sharing Trees) are tested on reachability problems of bounded PNs in GGZ95 ST99. As mentioned in the introduction, our heuristics could be incorporated, e.g., in a BDD-based framework. Our use of heuristics shares some similarities with depth-first search algorithms Hol88 Val98 for state enumeration, an approach used to compute an under-approximation of the reachability graph. In fact, our heuristics gives us conditions to detect acyclic paths of the reachability graph that go from the initial marking to the current marking. However, note that the use of Parikh vectors allows us to check the absence of cycles on collections of paths (all paths and related subpaths represented by the vector). Furthermore, the use of the second heuristics allows us to obtain more accurate information w.r.t. a generic depth-first search where only the current paths is memoized. Depth-first search algorithms combined with methods for storing visited markings have been proposed in T.T91 MK96. As heuristics for garbage collection, in I.I91 Jard and Jéron propose to discharge states selected randomly from the set of visited markings, whereas Miller and Katz in MK96 select the states to discharge using their revisiting degree. Differently from J.T91 MK96, our method is based on a breadth-first search, in which we use the rank of minimal T-semiflows (i.e., heuristics peculiar of Petri Nets) to guide garbage collection. 


\section{Conclusions}

We have presented a new algorithm for validating concurrent systems modeled as bounded Petri Nets. Our method is combines forward state exploration with two structural heuristics based on the properties of T-semiflows. One of the main feature of our heuristics is that they give us an estimation on the number of levels of the reachability graph we need to keep in memory. Using this measure, we can organize main memory as a circular array, so as to garbage collect states outside the current working window. In our prototype, information for computing error traces are stored on disk. In this preliminary work, we were mainly interested in evaluating the applicability of the method (are there interesting examples that fulfill our assumptions?), and the efficacy of the specialized memory management (can we save memory?). In this respects, we think that our results are quite promising (see Section 5. For a better evaluation of the approach (e.g. to compare its scalability w.r.t. BDD-based approaches like MC99 PCP99), we plan to integrate efficient data structures within our preliminary naive implementation of the algorithm (in which vectors are stored as sequences of slots, as described in Section 5. Finally, it would be interesting to study the applicability of similar techniques for the validation of infinite-state systems, e.g., integrated in approaches like DR00.

Acknowledgements. The authors would like to thank Javier Esparza for having pointed out to us several important references, Jean-Francois Raskin for fruitful discussions, and the anonymous reviewers for useful suggestions and the pointers to J.191 MK96.

\section{References}

$\mathrm{ABC}^{+}$95. M. Ajmone Marsan, G. Balbo, G. Conte, S. Donatelli, and G. Franceschinis. Modelling with Generalized Stochastic Petri Nets. Series in Parallel Computing. John Wiley \& Sons, 1995.

$\mathrm{BCB}^{+} 90$. J. R. Burch, E. M. Clarke, K. L. McMillan, D. L. Dill, and J. Hwang. Symbolic Model Checking: $10^{20}$ States and Beyond. In Proc. LICS '90, pages 428-439, 1990.

Chi89. G. Chiola. Compiling Techniques for the Analysis of Stochastic Petri Nets. In Modelling Techniques and Tools for Computer Performance Evaluation, pages 11-24, 1989.

CFGR95. G. Chiola, G. Franceschinis, R. Gaeta, and M. Ribaudo. GreatSPN 1.7: Graphical Editor and Analyzer for Timed and Stochastic Petri Nets. In Performance Evaluation, 24(1-2):47-68, 1995.

CM97. G. Ciardo and A. S. Miner. Storage Alternatives for large structured state spaces. In Proc. Modelling Techniques and Tools for Computer Performance Evaluation, LNCS 1245, pages 44-57. Springer, 1997.

CDC00. R. Carvajal-Schiaffino, G. Delzanno, and G. Chiola. Combining Structural and Enumerative Techniques for the Validation of Bounded Petri Nets: A New 'Type 2' Validation Algorithm. Technical Report, DISI-00-10, Dipartimento di Informatica e Scienze dell'Informazione dell'Università di Genova, October 2000. 
DR00. G. Delzanno and J. F. Raskin. Symbolic Representation of Upward-closed Sets. In Proc. TACAS 2000, LNCS 1785, pages 426-440. Springer, 2000.

Des98. J. Desel. Basic Linear Algebraic Techniques for Place/Transition Nets. In Reisig and Rozenberg KR98, pages 257-308, 1998.

DE95. J. Desel and J. Esparza. Free Choice Petri Nets. Cambridge University Press, 1995.

DR98. J. Desel and W. Reisig. Place/Transition Petri Nets. In Reisig and Rozenberg RR98, pages 122-173, 1998.

EM00. J. Esparza and S. Melzer. Verification of safety properties using integer programming: Beyond the state equation. Formal Methods in System Design, $16: 159-189,2000$.

GGZ95. F. Gagnon, J.-Ch. Grégoire, and D. Zampuniéris. Sharing Trees for 'Onthe-fly' Verification. In Proc. FORTE '95, 1995.

Gra97. B. Grahlmann. The PEP Tool. In Proc. CAV'97, LNCS 1254, pages 440443. Springer, 1997.

Hol88. G. Holzmann. Algorithms for Automated Protocol Verification. AT\&T Technical Journal 69(2):32-44, 1988.

JJ91. C. Jard and Th. Jéron. Bounded-memory Algorithms. In Proc. CAV'91, LNCS 575, pages 192-202. Springer, 1991.

MCC97. P. Marenzoni, S. Caselli, and G. Conte. Analysis of Large GSPN Models: A Distributed Solution Tool. In Proc. Int. Work. on Petri Nets and Performance, 1997.

McM93. K. L. McMillan. Symbolic Model Checking: An Approach to the State Explosion Problem. Kluwer Academic, 1993.

MK96. H. Miller and S. Katz. Saving Space by Fully Exploiting Invisible Transitions. In Proc. CAV '96, LNCS 1102, pages 336-347. Springer, 1996.

MC99. A. Miner and G. Ciardo. Efficient Reachability Set Generation and Storage using Decision Diagrams. In Proc. ICATPN '99, LNCS 1639, pages 6-25. Springer, 1999.

PCP99. E. Pastor, J. Cortadella, and M. A. Peña. Structural Methods to Improve the Symbolic Analysis of Petri Nets. In Proc. ICATPN '99, LNCS 1639, pages 26-45. Springer, 1999.

Rei86. W. Reisig. Petri Nets. An introduction. EATCS Monographs on Theoretical Computer Science, Springer 1986.

RR98. W. Reisig and G. Rozenberg, editors. Lectures on Petri Nets I: Basic Models. Advances in Petri Nets, LNCS 1491. Springer, 1998.

Sch94. A. Schrijver. Theory of Linear and Integer Programming, Wiley \& Sons, 1994.

STC98. M. Silva, E. Teruel, and J. M. Colom. Linear Algebraic and Linear Programming Techniques for Analysis of Place/Transition Net Systems. In Reisig and Rozenberg RR98, pages 308-309, 1998.

ST99. K. Strehl and L. Thiele. Interval Diagram Techniques For Symbolic Model Checking of Petri Nets. In Proc. DATE'99, pages 756-757, 1999.

Val98. A. Valmari. The State Explosion Problem. In Reisig and Rozenberg RR98, pages 308-309, 1998.

Wim97. G. Wimmel. A BDD-based Model Checker for the PEP Tool. Technical Report, University of Newcastle upon Tyne, 1997. 